\title{
Controlling Calcium Carbonate Particle Morphology, Size, and Molecular Order Using Silicate
}

\author{
Lior Minkowicz $^{1}$, Arie Dagan ${ }^{1}$, Vladimir Uvarov ${ }^{2}$ and Ofra Benny ${ }^{1, *}$ \\ 1 The Institute for Drug Research, The School of Pharmacy, Faculty of Medicine, The Hebrew University of \\ Jerusalem, Jerusalem 91120, Israel; lior.minkowicz@mail.huji.ac.il (L.M.); arie.dagan@mail.huji.ac.il (A.D.) \\ 2 The Center for Nanoscience and Nanotechnology, The Faculty of Science, The Hebrew University of \\ Jerusalem, Jerusalem 91120, Israel; vladimiru@savion.huji.ac.il \\ * Correspondence: ofra.benny@mail.huji.ac.il
}

check for updates

Citation: Minkowicz, L.; Dagan, A.; Uvarov, V.; Benny, O. Controlling Calcium Carbonate Particle Morphology, Size, and Molecular Order Using Silicate. Materials 2021, 14, 3525. https://doi.org/10.3390/ ma14133525

Academic Editor: Fabien Delpech

Received: 11 May 2021

Accepted: 22 June 2021

Published: 24 June 2021

Publisher's Note: MDPI stays neutral with regard to jurisdictional claims in published maps and institutional affiliations.

Copyright: (c) 2021 by the authors. Licensee MDPI, Basel, Switzerland. This article is an open access article distributed under the terms and conditions of the Creative Commons Attribution (CC BY) license (https:// creativecommons.org/licenses/by/ $4.0 /)$.

\begin{abstract}
Calcium carbonate $\left(\mathrm{CaCO}_{3}\right)$ is one of the most abundant substances on earth and has a large array of industrial applications. Considerable research has been conducted in an effort to synthesize calcium carbonate microparticles with controllable and specific morphologies and sizes. $\mathrm{CaCO}_{3}$ produced by a precipitation reaction of calcium nitrate and sodium carbonate solution was found to have high polymorphism and batch to batch variability. In this study, we investigated the polymorphism of the precipitated material and analyzed the chemical composition, particle morphology, and crystalline state revealing that the presence of silicon atoms in the precipitant is a key factor effecting particle shape and crystal state. An elemental analysis of single particles within a polymorphic sample, using energy-dispersive X-ray spectroscopy (EDS) conjugated microscopy, showed that only spherical particles, but not irregular shaped one, contained traces of silicon atoms. In agreement, silicon-containing additives lead to homogenous, amorphous nanosphere particles, verified by X-ray powder diffraction (XRD). Our findings provide important insights into the mechanism of calcium carbonate synthesis, as well as introducing a method to control the precipitants at the micro-scale for many diverse applications.
\end{abstract}

Keywords: calcium carbonate; sodium silicate; calcite; EDS; nanoparticles

\section{Introduction}

Calcium carbonate $\left(\mathrm{CaCO}_{3}\right)$ is one of the most abundant substances on earth. This mineral is found naturally in rocks and is the main component of pearls and of shells of marine organisms. Calcium carbonate has three main crystalline states, calcite, vaterite, and aragonite, which each having typical morphology rhombohedral, spherical, and needlelike, respectively [1]. This mineral has many utilizations; it is a main active ingredient in agricultural lime [2], it is being used as a filler material in the paper and colors industries, and is widely used as building material in the construction industry [3,4]. In medicine, it is used as an inexpensive dietary calcium supplement [5,6], an anti-acid, [7], and an inert filler for tablets and other pharmaceuticals [8]. Due to its positive charge and erosion capability, calcium carbonate has also been explored preclinically as a template for layer-by-layer (LbL) deposition in drug delivery applications [9-11]. As with any particulate pharmaceutical product, the ability to control the physicochemical properties of the product is a critical issue as particle size, shape, and composition dictate the drug's performance, e.g., biodistribution, selectivity, and elimination from the body. Despite remarkable advantages for calcium carbonate, including low cost and high biocompatibility, its utilization in biomedicine is limited by the low reproducibility of the synthesized end-product and high polymorphism that make the control over particle morphology very challenging. Therefore, a key task in this regard would be to identify which factors affect the synthesis and to correlate between material composition and morphology, thus providing a predictable product with well-defined properties. 
In this study, we attempted first to evaluate the reproducibility of the synthesis reaction and identify what components affect the morphology of the end-product. We therefore performed microscopical morphology study followed by chemical analysis in order to identify these potential effectors. The calcium carbonate particles were obtained by precipitation reaction using soluble calcium nitrate and sodium carbonate as previously described $[1,12,13]$ with or without the addition of carboxymethylcellulose (CMC) [12], which was previously shown to affect particle shape [14]. Surprisingly, we found that despite repeating the exact same protocol, the product was still highly polymorphic and had wide batch-to-batch variability. By analyzing the product using energy-dispersive $X$ ray spectroscopy (EDS) we revealed that only particles carrying spherical shape contained residual silicon atoms $(<1 \%)$, while non-spherical particles had no detectable silicon atoms. To further validate our findings, we repeated the original precipitation reaction in the presence of silicon-containing additives added exogenously. Different silicate additives were explored, revealing that the presence of silicate shifts the synthesis towards the production of spherical nanoparticles in a concentration-dependent manner. Moreover, we found that the contained silicate preserved the amorphous state of the mineral, while in its absence, the mineral was in its crystalline, calcite, state. Various concentrations of soluble silicate produced nanospheres with range of silicon atom content, measured by inductively-coupled plasma optical emission spectroscopy (ICP-OES). Further studies are required to determine the exact mechanism of crystallization and the role of silicate in this reaction. The ramifications of the current work are the possibility of being able to better control the morphology of silicon-containing calcium carbonate composite particles and as a result increasing its use in biomedicine in the form of nanoparticles.

\section{Experimental Methodology}

2.1. Preparation of Calcium Carbonate $\left(\mathrm{CaCO}_{3}\right)$ Particles

Calcium carbonate particles were formed by a chemical reaction between $\mathrm{CO}_{3}{ }^{2-}$ and $\mathrm{Ca}^{2+}$ as described elsewhere [12]. The following chemical reaction was performed, with or without CMC (Formula (1)):

$$
\mathrm{Na}_{2} \mathrm{CO}_{3}+\mathrm{Ca}\left(\mathrm{NO}_{3}\right)_{2} \rightarrow \mathrm{CaCO}_{3(\mathrm{~s})}+2 \mathrm{Na}^{+}+2 \mathrm{NO}_{3}{ }^{-}
$$

The basic protocol used throughout the study is detailed below and any variation is mentioned specifically in the text. Sodium carbonate solution (0.025 M; Sigma, S2127, St. Louis, MO, USA) was mixed with 0.17\% w/v CMC (Fluka, BioChemika, 21901, ultra-low viscosity, 20-50 mPa.s) in a volume of $25 \mathrm{~mL}$ DDW and sonicated for $1 \mathrm{~min}$. A solution of $0.025 \mathrm{M}(25 \mathrm{~mL})$ calcium nitrate in DDW (Sigma, Steinheim, Germany \#31218) was added and the $\mathrm{CaCO}_{3}$ was allowed to precipitate under a magnetic stirrer for $5 \mathrm{~min}$. The product was then centrifuged for $5 \mathrm{~min}$ at $1000 \times \mathrm{g}$. The precipitate was washed twice with DDW and lyophilized for $48 \mathrm{~h}$. Sodium silicate solution in a density of $1.4 \mathrm{~g} / \mathrm{mL}$ (Sigma, St. Louis, MO, USA, \#338443) was added to the sodium carbonate solution or, alternatively, was mixed together with the calcium nitrate.

\subsection{Characterization of Crystal State of $\mathrm{CaCO}_{3}$ Particles by Powder X-ray Diffraction}

The crystalline structure of the specimens was analyzed by XRD. Measurements were performed using a D8 Advance diffractometer (Bruker AXS, Karlsruhe, Germany). A low background quartz sample holder was carefully filled with the powder samples. The $X R D$ patterns from 5 to $70^{\circ} 2 \theta$ were recorded at room temperature using $\mathrm{CuKa}$ radiation $(\lambda=0.15418 \mathrm{~nm})$ with the following measurement conditions: Tube voltage of $40 \mathrm{kV}$, tube current of $40 \mathrm{~mA}$, step scan mode with a step size $0.02^{\circ} 2 \theta$, and counting time of $1 \mathrm{~s}$ per step. Phase identification and quantification were performed using EVA software (Bruker AXS).

\subsection{Morphological Studies Using Electron Microscopy}

Morphology was observed and chemical composition was identified with the environmental Scanning Electron Microscope (SEM) Quanta 200 (FEI Company, The Netherlands), 
equipped with an energy-dispersive X-ray spectroscopy detector (EDAX-TSL, AMETEK, Hillsboro, OR, USA). For SEM analysis and imaging, the particles were sampled on a conductive adhesive tape, metal coated with a thin film of Pd/Au sputtered onto the sample (SC7620 Sputter coater, East Sussex, UK) and air-dried. Some of the samples were subjected to chemical elemental analysis with an EDS function. The elemental composition of a sample was determined using a characteristic $X$-ray spectrum. The analysis was performed either in a frame mode, which sums the elemental composition of the particles, or in a spot mode, in which the beam targets a single area chosen within the field of view that facilitates the characterization of various particles in a single sample.

Transmission electron microscopy (TEM) observations were carried out with Jeol, TEM 1400Plus, Japan, with a charge-coupled camera Gatan Orius SC600 to detect nanoparticles. Particles were stained with $2 \%$ uranyl acetate; the observation was subsequently made in a carbon film.

\subsection{Inductively-Coupled Plasma Optical Emission Spectroscopy}

ICP-OES quantitative elemental analysis was performed using PQ 9000 Elite (Analitik, Jena, Germany). Calibration was done with MERCK solution and accuracy confirmations were performed according to the standards of the US Geological Survey (USGS). Powder formulations were dissolved in $50 \mathrm{~mL}$ DDW in $1 \%$ nitric acid $\left(\mathrm{HNO}_{3}\right)$ before elemental analysis.

\section{Results}

\subsection{Analyzing Morphology and Composition of Calcium Carbonate Samples}

Production of calcium carbonate particles in a reproducible manner and with a predictable shape is highly challenging and standard protocols would often yield high sample variability. Elucidating the parameters that affecting calcium carbonate particles can greatly contribute to the establishment of robust synthesis protocols. Producing uniform products at the micro or nanometric scales are conditional step toward expanding the utilization of calcium carbonate particles in the high standard required in biomedicine.

To assess calcium carbonate polymorphism and determine the variability of the reactions we first followed standard protocols using sodium carbonate, $\mathrm{CMC}$, and calcium nitrate [12]. Repeating the exact procedure multiple times revealed high batch to batch variability as shown with SEM imaging in Figure 1 (representative images of three independent batches). In each batch particles acquired entirely different morphologies of spheres, cubes, and ellipsoids. We attempted to control particle formation by erosion by adding calcium chelator $(0.25 \mu \mathrm{M}$ ethylenediaminetetraacetic acid (EDTA)) to the synthesis reaction for various durations $(2,5$, and $15 \mathrm{~min})$. However, there was no advantage over product uniformity and the sample contained diverse product with size range of $\sim 1-5 \mu \mathrm{m}$ (Figure S1).

In order to determine the origin of the polymorphism in samples, we performed a composition EDS/SEM analysis for spherical particles, in comparison to the non-spherical crystals at the same sample. As shown in Figure 2 and Figure S2, we consistently found that only spheres, and not other shapes, contained a very low $(<1 \%)$ amount of silicon atoms in addition to the other expected elements. The presence of gold traces was the result of sample coating prior to SEM analysis. Based on this unexpected observation, we attempted to determine whether silicon-containing additives may shift the morphology of the calcium carbonate towards specific spherical morphology. Therefore, we repeated the reaction in the presence of various silicon-containing additives. 
A

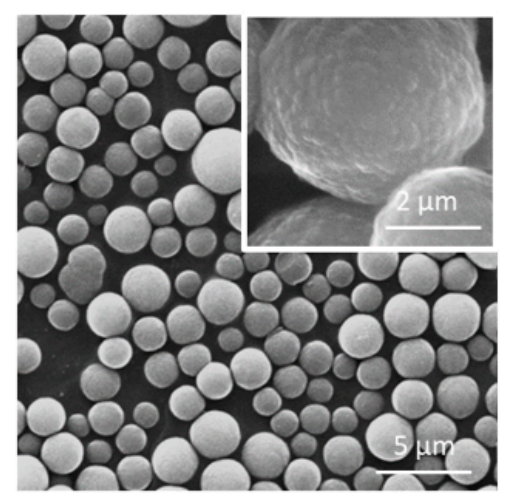

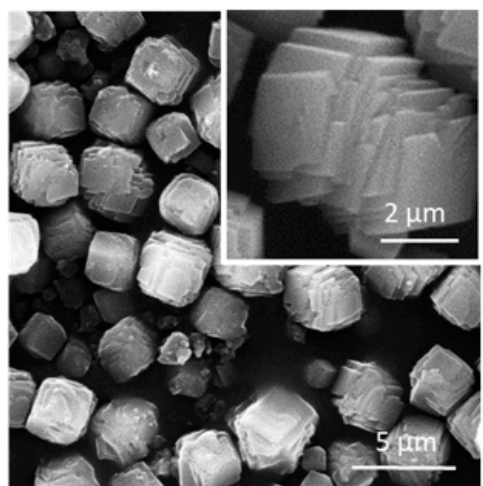

c

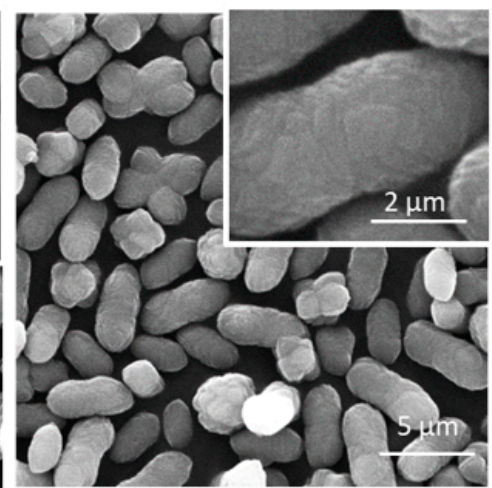

Figure 1. Calcium carbonate fabricated with CMC produces polymorphic samples and heterogenic batches. SEM images (A-C) from three independent samples, fabricated with the same standard protocol, show high batch to batch variation. Magnification $\times 10,000$.

A

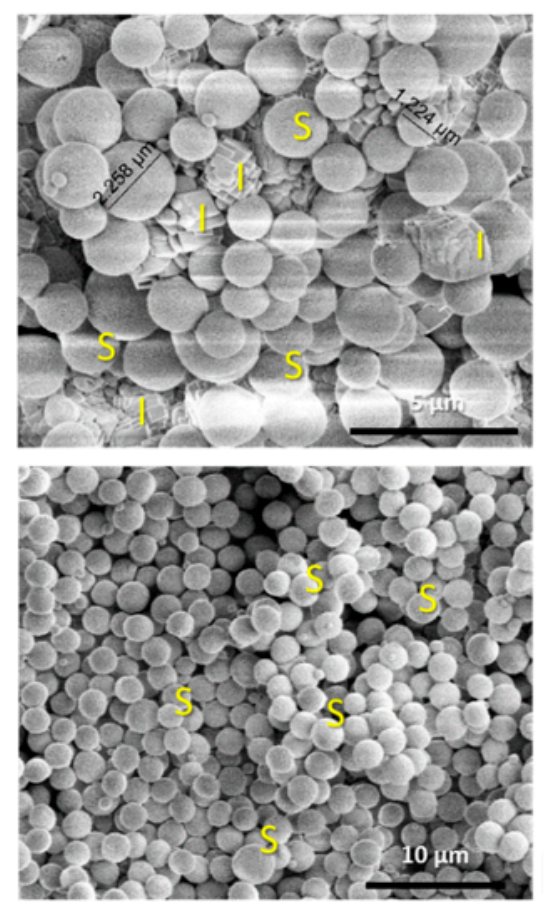

B
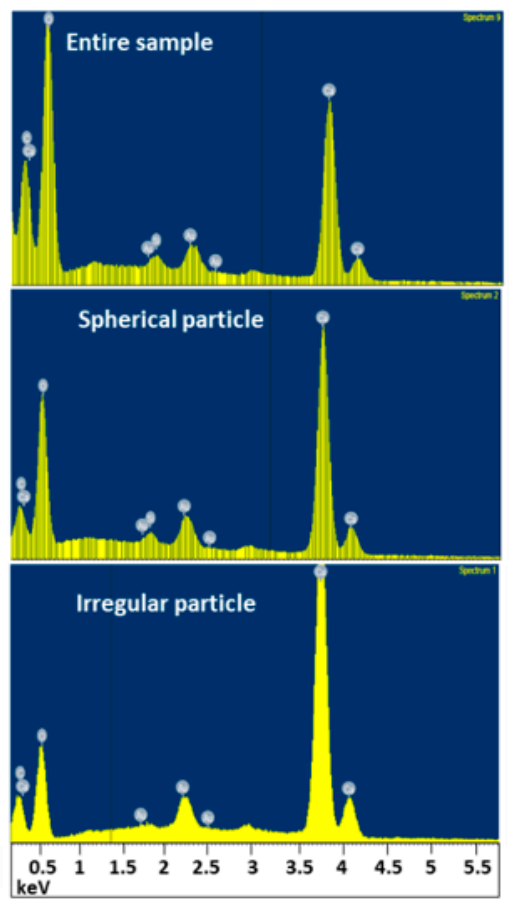

C

\begin{tabular}{|c|c|}
\hline Element & Atomic\% \\
\hline C & 22 \\
\hline O & 60 \\
\hline Ca & 17 \\
\hline Au & 1 \\
\hline Element & Atomic\% \\
\hline C & 10 \\
\hline O & 58 \\
\hline Si & 1 \\
\hline Ca & 30 \\
\hline Au & 1 \\
\hline Element & Atomic\% \\
\hline C & 11 \\
\hline O & 45 \\
\hline Au & 43 \\
\hline
\end{tabular}

Figure 2. Silicon element found in sphere particles after EDS elemental analysis of $\mathrm{CaCO}_{3}$ with $\mathrm{CMC}$ (sample $1 \mathrm{~A}$ ). The analysis includes the overall sample, single spheres, and single irregular shaped particles. (A) SEM images of the particles which were targeted by EDS. (B,C) Elemental analysis of the overall sample and targeted particles. Silicon was found in the entire sample and in spherical particles.

\subsection{Addition of Silicon-Containing Additives and their Effect on Calcium Carbonate Particles}

Commercial additives-silicon grease and silicone gel-that contain a mixture of silicate molecules in an undefined composition were dispersed or solubilized in organic solvents and were added to the precipitation reaction of calcium carbonate, as shown in (Figure S3). In the presence of silicone grease dissolved in ethyl acetate (EtOAc), small, uniform microparticles were obtained (Figures S3E and S4). Analysis with EDS showed, in agreement to our initial observation, that traces of silicon atoms were present in spheres but not in cubes. Figure S5 summarizes the morphology data obtained with the various silicon-containing additives. 
In order to study the effect of specific and defined silicon-containing molecules on calcium carbonate synthesis, we selected a soluble form of silicon compound, i.e., sodium silicate. Commercial sodium silicate solution at a concentration of $1 \% v / v$ was added to the synthesis, and particle morphology was studied (Figure 3). In the presence of silicate, the particles were smaller than previously obtained with nanoparticles size range of 30 to $50 \mathrm{~nm}$ with larger particles of $\sim 200-250 \mathrm{~nm}$ (Figure 3B).

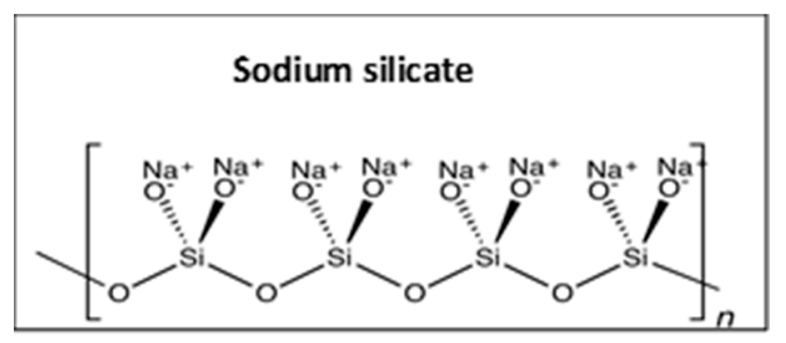

(A)
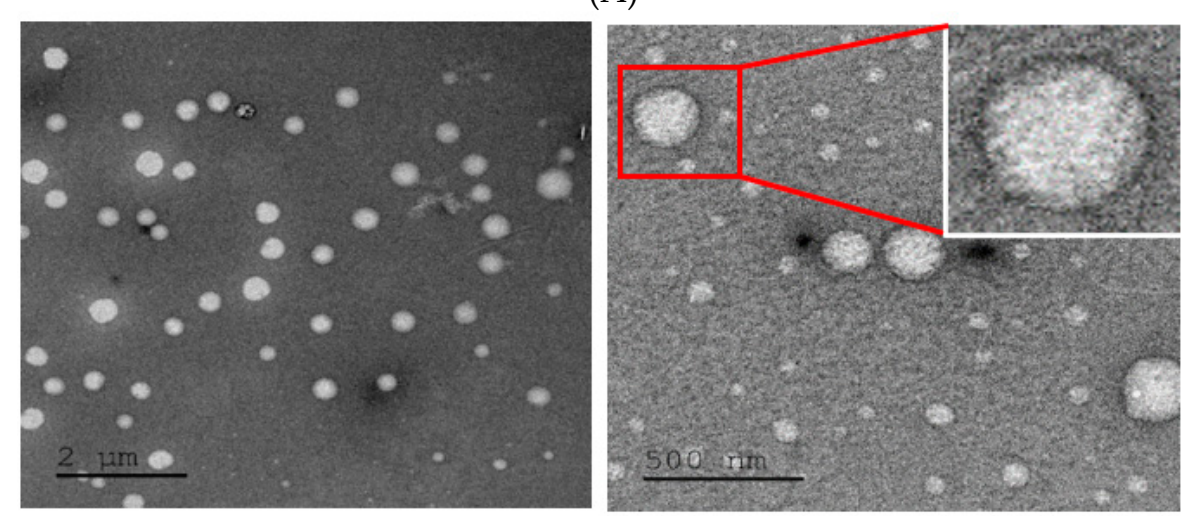

(B)

Figure 3. Effect of sodium silicate on particle size and shape. (A) Chemical structure of sodium silicate. (B) TEM images of particles fabricated with sodium silicate and CMC showing nanospheres with a size range of 30 to $50 \mathrm{~nm}$ together with some larger particles $\sim 200-250 \mathrm{~nm}$.

\subsection{Crystalline State of Calcium Carbonate with Various Content of Silicate}

The crystalline state of the material was determined by $X$-ray powder diffraction (XRD).

The results showed that silicate containing products maintained their amorphous state of the material, while calcite particles were formed in the absent of silicate (Figure 4A,B). Therewith, calcite was found in pure form when particles were synthesized without sodium silicate, and the mean crystallite size of calcite calculated using the Scherrer equation [15] was $\sim 40 \mathrm{~nm}$. To eliminate the possibility that the basic $\mathrm{pH}$ of the silicate solvent affected particle state rather than the silicate itself, we repeated the reaction in the presence of $0.1 \% v / v \mathrm{NaOH}$ in water at the same concentrations as in the silicate solution. As can be seen in Figure 4C, we confirmed that $\mathrm{pH}$ had no effect on the morphology of calcium carbonate particles.

Importantly, the crystal state of the material was verified with XRD for varying silicate contents (Figure 5A). It was found to be a mixture of calcite and vaterite with approximate ratio 20:80. The crystal size of calcite and vaterite calculated using the Scherrer equation was $87 \mathrm{~nm}$ and $22 \mathrm{~nm}$, respectively. The crystal state of the material was verified with XRD for varying silicate contents and quantitative analysis of samples with increasing amounts of sodium silicate was performed using ICP-OES. The analysis showed that 0.05 and $0.1 \mathrm{~mL}$ of sodium silicate resulted in atomic ratios of $\sim 1: 4$ and $\sim$ 1:2 for silicon:calcium (Figure $5 B$ ). 

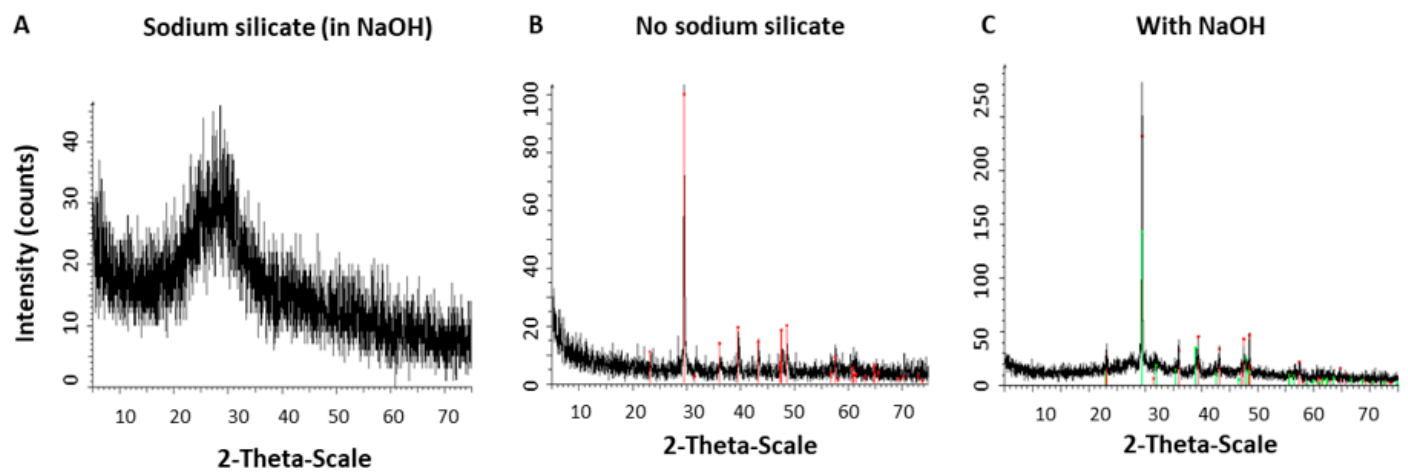

Figure 4. Effect of sodium silicate on the crystal state of the material. (A,B) XRD analysis of the particles synthesized with sodium silicate resulted in an amorphous material, while absence of silicate produced the high ordered form of the material, calcite. (C) Control, only the solvent of silicate solution was added $(\mathrm{NaOH})$ showing no effect on the material's crystalline state.

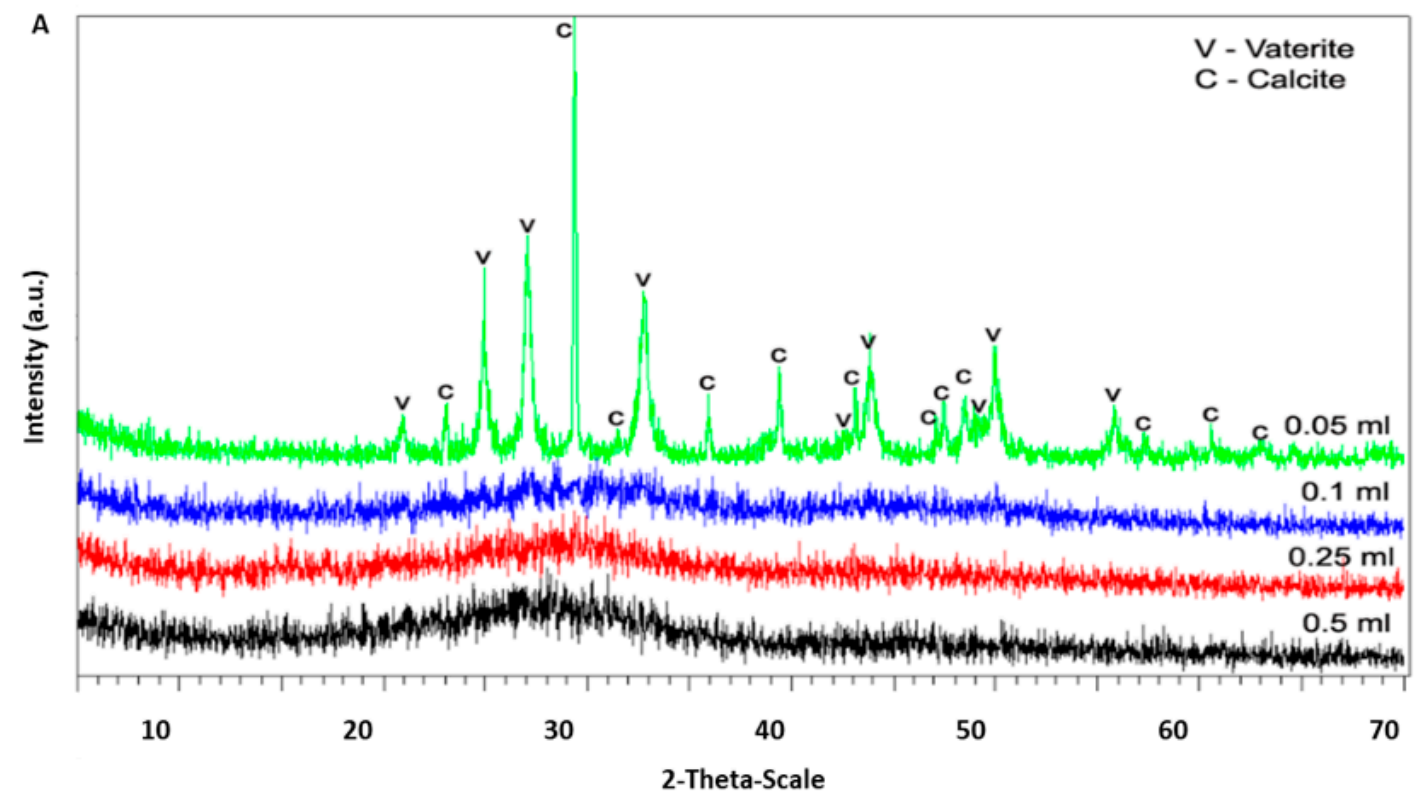

\begin{tabular}{|c|c|c|}
\hline Formulation & $\mathrm{Si}(\mathrm{mg} / \mathrm{L})$ & $\mathrm{Ca}(\mathrm{mg} / \mathrm{L})$ \\
\hline Calcium carbonate & $<0.1$ & 135.2 \\
\hline Calcium carbonate- $0.05 \mathrm{ml}$ sodium silicate & 36.0 & 133 \\
\hline Calcium carbonate- $0.1 \mathrm{ml}$ sodium silicate & 103.4 & 211 \\
\hline
\end{tabular}

Figure 5. Calcium carbonate synthesized with elevated concentrations of sodium silicate. Decreasing sodium silicate concentration from $0.5 \mathrm{~mL}(1 \% \mathrm{v} / \mathrm{v})$ to $0.05 \mathrm{~mL}$ resulted in a shift from amorphous material (high silicate) to calcite (no silicate) as assessed by XRD graphs (A) and numeral data (B).

\subsection{The Effect of CMC and Product Stability}

Repeating the synthesis in the presence of silicate but without CMC yielded similar results to the CMC-containing samples, suggesting that CMC does not have a critical role in the formation of nanospheres (Figure 6). Importantly, the material was found to be stable over two weeks without apparent visual changes (Figure 7A), and the reaction was unaffected by high temperature as both $4{ }^{\circ} \mathrm{C}$ and $45^{\circ} \mathrm{C}$ reactions produced similar products in terms of size and morphology (Figure 7B). 

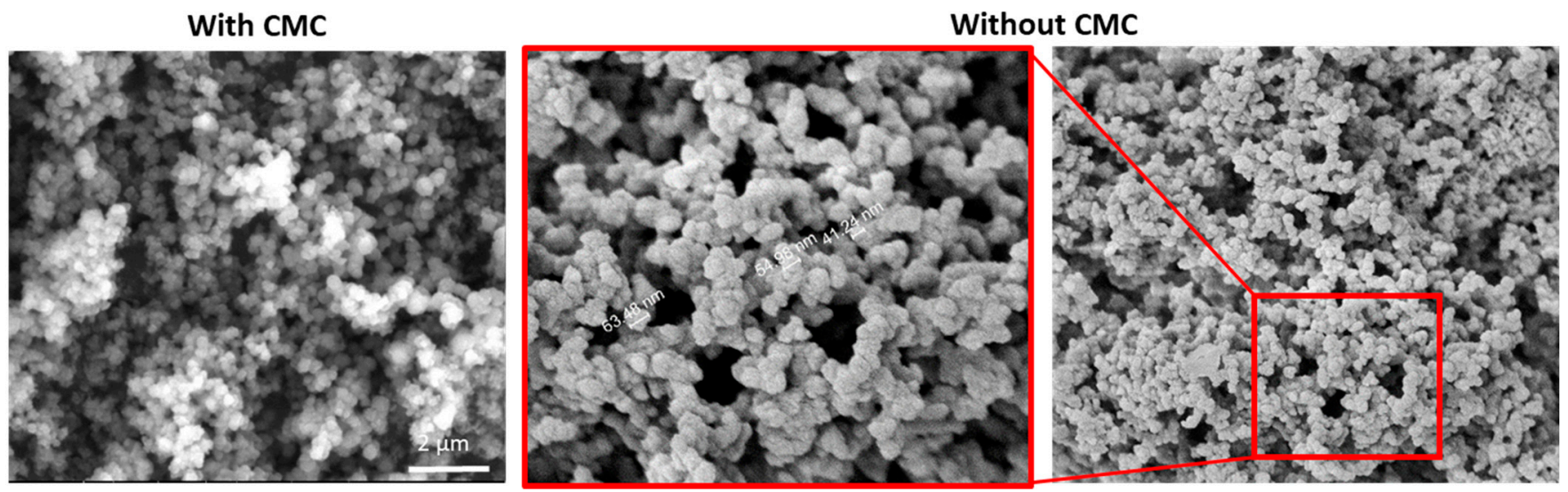

Figure 6. High-resolution SEM images of calcium carbonate particles produced with and without CMC. Nanospheres were obtained with the presence of silicate. Similar morphologies were obtained with or without the presence of CMC.

A

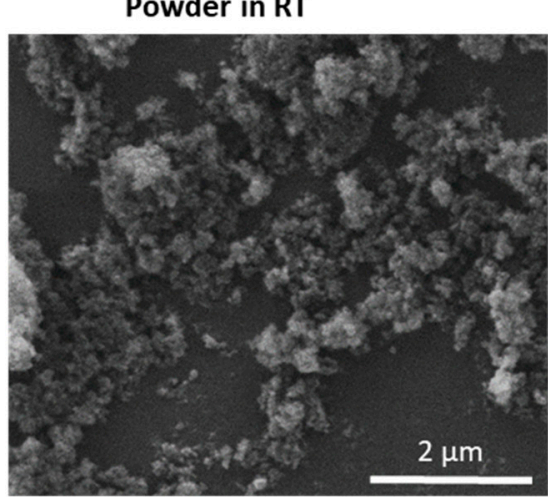

B $4^{\circ} \mathrm{C}$

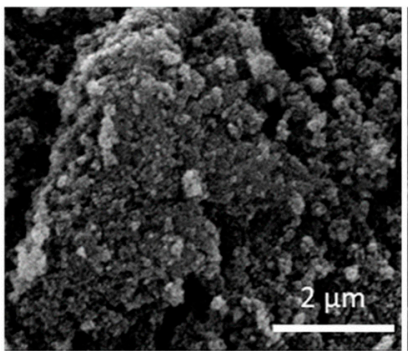

Suspension in RT

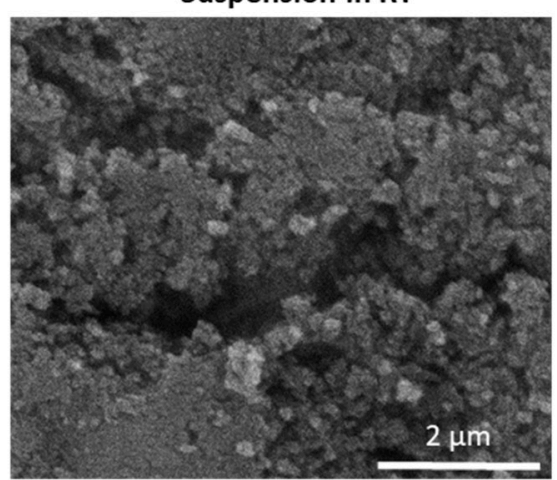

\section{Suspension in $4^{\circ} \mathrm{C}$}

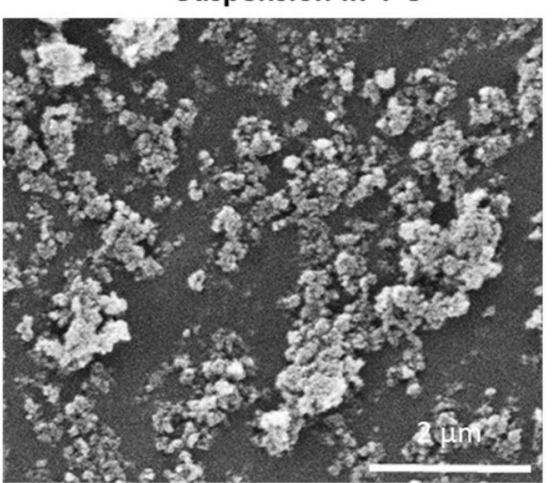

$45^{\circ} \mathrm{C}$
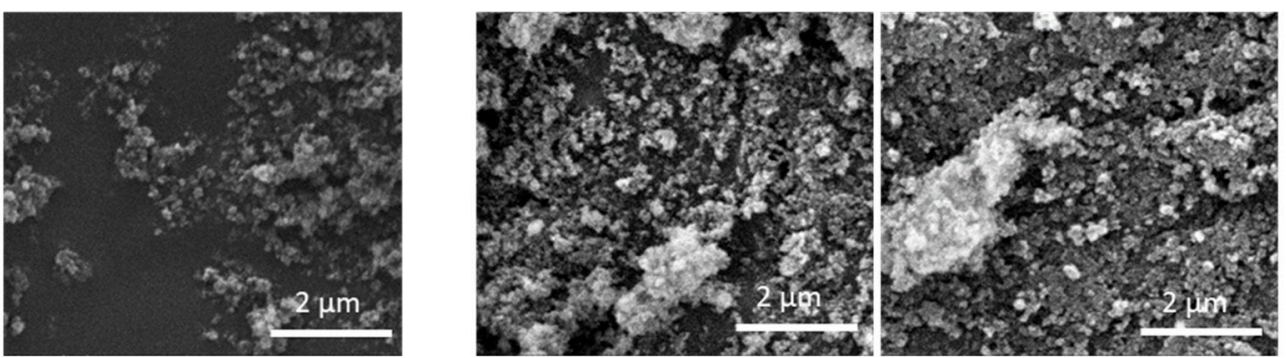

Figure 7. Stability of Calcium carbonate-silicate in different storage conditions and temperature. (A) SEM images of samples that were stored as a powder in room temperature; suspension in room temperature; or as a suspension in $2-8^{\circ} \mathrm{C}$. (B) SEM images of synthesized in $4{ }^{\circ} \mathrm{C}$ or in $45^{\circ} \mathrm{C}$.

\section{Discussion}

Calcium carbonate particles are widely used in various industrial and biotechnological applications due to their beneficial properties such as high porosity, non-toxicity, and biocompatibility $[8,16,17]$. This polymorphic compound has various structural states, of which the amorphous calcium carbonate is relatively short-lived and unstable and is thought to act as a seed for the crystal growth of the other three polymorph crystals. These crystals, namely, calcite, vaterite, and aragonite, have, respectively, rhombohedral, spherical, and needle-like morphologies [1]. The transition between the different states is a topic of much research. Several reports concerning calcium carbonate have described spheres, cubes, and other shapes, such as ellipsoids and discs $[1,12,13]$. However, in most cases, the reproducibility of the synthesis, as well as the key reaction parameters that govern the shape of the particles, were not reported. 
Many efforts to improve reproducibility and control the calcium carbonate properties included the addition of various excipients to the precipitation reaction. When used as a pigment material, the addition of CMC was shown to affect calcium carbonate particle morphology, improve optical performance, and reduce particle mechanical strength, which can be further improved by supplementation with corn starch [18]. In another study, CMC was shown to induce spherical particle formation [14] and contribute negative charges, thus making the particles suitable as templates for LbL deposition [12]. In our experiments, using CMC as a single additive revealed that the presence of silicate was the critical factor for producing nanospheres and not the CMC itself as similar nanospheres were obtained with or without the CMC as shown in Figure 6. Additional studies used additives that affected particle morphology and size. For example, Yu et al. showed that the addition of polyacrylic acid induced the formation of cubic monodispersed calcite particles, emphasizing the importance of a single additive in the synthesis process on particle morphology [19]. Similarly, it was shown that EDTA triggers the formation of spherical particles, while citrate shifts the reaction to form mainly rhombic particles [20]. In our study, we could not detect a clear effect of EDTA on the product.

In drug delivery, nano- and microparticles are often being utilized as carriers with superior pharmacological benefits comparing to free drugs [21,22]. In this respect, calcium carbonate may be useful due to its biocompatibility and low cost. Particle biophysical properties affect drug biodistribution, selective targeting, and elimination from the body [23-25]. Therefore, extensive efforts have been invested in controlling the fabrication of particles, either as drug carriers or as templates for LbL formulations. The ability to reproduce the production of calcium carbonate was determined by repeating the precipitation reaction for several times and characterize the end-product. The results indicated that the product is highly polymorphic and carries wide batch-to-batch variability. Analysis with EDS/SEM, enabled to detect traces of the element silicon but only in the spherical particles. This observation strongly suggests that the presence of silicon affects the production of spherical shaped particles. One of the proposed explanations is that silicon stabilizes the amorphous state of the material, as has been suggested in regard to other molecules. Aizenberg et al. [26] showed that the presence of magnesium and macromolecules extracted from different biogenic sources shifted the synthesis of calcium carbonate from calcite to amorphous calcium carbonate and/or inhibited crystallization in vitro. In agreement with these observations, we found that silicate shifted the synthesis of calcium carbonate to an amorphous state.

Many previous reports that described the production of spherical calcium carbonate might have also contained unintentional silicon traces, possibly due to contamination from glassware or other sources. To establish a robust protocol that can be repeated by others, silicon must be supplemented exogenously to the reaction in a controlled manner. Therefore, we used different sources of silicon-containing materials, some insoluble (silica gel and grease) and some soluble (dissolved grease in ethyl acetate/sodium silicate solution). Out of all additives tested, we found that dispersed silica grease in ethyl acetate produced the most uniform microspheres, and interestingly, the addition of soluble sodium silicate produced smaller particles at the nano range. Further analysis of the thermostatic state of the nanospheres revealed that the material remained in its amorphous state and did not crystallize. We assume that the presence of silicon in the sample might stabilize the amorphic state of the material as the crystal begins to form at the submicroscopic level, possibly acting partly as an antiscalant for calcium carbonate, ref. [27] inhibiting the formation of the crystallized mineral and maintaining it at a nanoscale and in an amorphic state.

Quantitative analysis of the nanospheres (Figure 5) showed that doubling the sodium silicate content increased the silicon:calcium ratio in the particles (from $\sim 1: 2$ to $\sim 1: 4$ ).

\section{Conclusions}

Precipitation of calcium carbonate is an environmental sensitive reaction with delicate process that reduce the capacity to control the end product. There is a high innate poly- 
morphism in a given sample as well as high batch to batch difference. Our study revealed that the present of silicon in the precipitants affect the morphology of the mineral thus producing spherical and amorphous material. The synthesis of calcium carbonate was highly affected by to the presence of silicates residues and the exact composition of the additive affect the nature of the end product in terms of shape and size. Finally, we concluded that it is possible to control the morphology of calcium carbonate and it size where a minimal ratio of 1:4 silicon: Calcium is required to yield nanosphere-shaped particles. The capacity to control the production of the material is expected to impact substantially its potential usage in various fields including biomedicine.

Supplementary Materials: The following are available online at https://www.mdpi.com/article/10

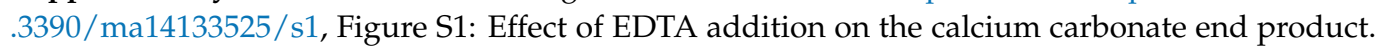
EDTA, $50 \mu \mathrm{L}$ of $0.25 \mathrm{M}$ was added and stirred for designated periods of time, 2, 5 and $15 \mathrm{~min}$. Representative images of dry samples were taken by SEM. The morphology of the precipitated particles is shown with and without EDTA addition. Samples without EDTA were stirred for $2 \mathrm{~min}$ resulting in heterogenic samples containing various particle shapes. Bar $=5$ micron; Figure S2: Silicon element found in sphere particles and not in irregular shaped-particles following EDS elemental analysis of $\mathrm{CaCO}_{3}$. EDS chemical elemental analysis of a selected sample containing microspheres and particles with other shapes. The analyses are of the overall sample, single spheres and single irregular shaped particles. Elemental analysis of the entire sample as well as the specifically shaped particles is provided. Small amounts of silicon atoms were found in the entire sample analysis and in the spherical particles' analysis, while no silicon was detected in non-spherical particles. Gold element $(\mathrm{Au})$ detected is due to sample coating prior to SEM imaging; Figure S3: Effect of silicon containing additives-silica gel and silicone grease in the fabrication of particles. (A) Chemical structures of the mix additives used to fabricate the particles. (B) Control particles fabricated without any additives. (C) Particles fabricated with excess of silica gel (Silica dioxide). (D) Particles fabricated with excess of silicone grease. (E) Silicone grease was dissolved in EtOAc $(5 \% w / v)$ and used $0.1 \mathrm{~mL}$ with CMC in the synthesis reaction. Particles fabricated this way had a smaller diameter and were mostly spherical. Bar $=5$ micron; Figure S4: SEM and EDS analysis of particles fabricated with silicone grease and CMC. A sample of fabricated particles obtained with the same procedure using excess of silicone grease and $25 \mathrm{mg}$ of CMC. (A) SEM images of the particles that were analyzed in EDS. Cubes and spheres are present the sample. $(B, C)$ EDS analysis of the entire sample, the spheres and the irregular shaped particles. Silicon was found when analyzing the entire sample or individual sphere particles, while no silicone was found in the irregular shapes. Sodium (Na) traces found are due to the sodium carbonate reagent; Figure S5: Summary of the different particle types observed using different silicon containing additives. Calcium carbonate synthesized without additives resulted in various morphological shapes, size ranges of 1-3 microns. Addition of silicone grease to the synthesis environment resulted in particles of the same size range but with a larger fraction of spherical shapes. Dissolving the silicone grease in EtOAc resulted in particles of a smaller diameter, $0.3-1$ microns. Synthesis with sodium silicate reduced the particles' size further to 30-250 nanometers.

Author Contributions: Conceptualization, O.B., L.M., A.D.; methodology, L.M., V.U. and A.D.; formal analysis, L.M. and O.B.; investigation, O.B. and L.M.; resources, O.B.; data curation, L.M. and O.B.; writing—original draft preparation, L.M., V.U. and O.B.; writing—review and editing, L.M., V.U. and O.B.; supervision, O.B., funding acquisition, O.B. All authors have read and agreed to the published version of the manuscript.

Funding: This research received no external funding.

Institutional Review Board Statement: Not applicable.

Informed Consent Statement: Not applicable.

Data Availability Statement: Data sharing not applicable.

Acknowledgments: The authors thank Eliana Steinberg, Bella Shusterman and Riva Nataf for proofreading, Eduard Berenshtein from the Core Research Facility of The Hebrew University of Jerusalem for his help in the SEM and TEM studies, and Evgenia Blayvas from the Nano-center of The Hebrew University of Jerusalem for her help in the SEM studies. We also thank Center Bloom's Pharmacy at HUJI. 
Conflicts of Interest: The authors declare no conflict of interest.

\section{References}

1. Boyjoo, Y.; Pareek, V.K.; Liu, J. Synthesis of Micro and Nano-Sized Calcium Carbonate Particles and Their Applications. J. Mater. Chem. A 2014, 7, 14270-14288. [CrossRef]

2. Chan, K.Y.; Heenan, D.P. Effect of Lime $\left(\mathrm{CaCO}_{3}\right)$ Application on Soil Structural Stability of a Red Earth. Aust. J. Soil Res. 1998, 36, 73-86. [CrossRef]

3. Sang, H.C.; Jin, K.P.; Seung, K.L.; Sung, M.J.; Im, H.K.; Ji, W.A.; Hwan, K. Synthesis of Precipitated Calcium Carbonate Using a Limestone and Its Application in Paper Filler and Coating Color. Mater. Sci. Forum 2007, 10, 881-884.

4. Matschei, T.; Lothenbach, B.; Glasser, F.P. The Role of Calcium Carbonate in Cement Hydration. Cem. Concr. Res. 2007, 37, 551-558. [CrossRef]

5. Nicar, M.J.; Pak, C.Y.C. Calcium Bioavailability from Calcium Carbonate Andcalcium Citrate. J. Clin. Endocrinol. Metab. 1985, 61, 391-393. [CrossRef]

6. Mortensen, L.; Charles, P. Bioavailability of Calcium Supplements and the Effect of Vitamin D: Comparisons between Milk, Calcium Carbonate, and Calcium Carbonate plus Vitamin D. Am. J. Clin. Nutr. 1996, 63, 354-357. [CrossRef]

7. Decktor, D.L.; Robinson, M.; Maton, P.N.; Lanza, F.L.; Gottlieb, S. Effects of Aluminum/Magnesium Hydroxide and Calcium Carbonate on Esophageal and Gastric pH in Subjects with Heartburn. Am. J. Ther. 1995, 2, 546-552. [CrossRef]

8. Peck, G.E.; Soh, J.L.P.; Morris, K.R.; Augsburger, L.L.; Hoag, S.W.; Lachman, L.; Lieberman, H.A.; Schwartz, J.B. Pharmaceutical Dosage Forms; Informa Healthcare: Boca Raton, FL, USA, 2008; Volume 3. [CrossRef]

9. De Villiers, M.M.; Otto, D.P.; Lvov, Y.M. Application of Electrostatic Layer-by-Layer Nanocoating in Drug Delivery. Am. Pharm. Rev. 2012, 3, 2304.

10. Zhao, Y.; Luo, Z.; Li, M.; Qu, Q.; Ma, X.; Yu, S.H.; Zhao, Y. A Preloaded Amorphous Calcium Carbonate/doxorubicin@silica Nanoreactor for pH-Responsive Delivery of an Anticancer Drug. Angew. Chem. Int. Ed. 2015, 54, 919-922. [CrossRef]

11. Sukhorukov, G.B.; Volodkin, D.V.; Günther, A.M.; Petrov, A.I.; Shenoy, D.B.; Möhwald, H. Porous Calcium Carbonate Microparticles as Templates for Encapsulation of Bioactive Compounds. J. Mater. Chem. 2004, 2073-2081. [CrossRef]

12. Zhao, Q.; Mao, Z.; Gao, C.; Shen, J. Assembly of Multilayer Microcapsules on $\mathrm{CaCO}_{3}$ particles from Biocompatible Polysaccharides. J. Biomater. Sci. Polym. Ed. 2006, 17, 997-1014. [CrossRef]

13. Abebe, M.; Hedin, N.; Bacsik, Z. Spherical and Porous Particles of Calcium Carbonate Synthesized with Food Friendly Polymer Additives. Cryst. Growth Des. 2015, 15, 3609-3616. [CrossRef]

14. Klemm, D.; Heublein, B.; Fink, H.P.; Bohn, A. Cellulose: Fascinating Biopolymer and Sustainable Raw Material. Angew. Chem. Int. Ed. 2005, 3358-3393. [CrossRef]

15. Patterson, A.L. The Scherrer Formula for X-Ray Particle Size Determination. Phys. Rev. 1939, 56, 978-982. [CrossRef]

16. Zhang, Y.; Ma, P.; Wang, Y.; Du, J.; Zhou, Q.; Zhu, Z.; Yang, X.; Yuan, J. Biocompatibility of Porous Spherical Calcium Carbonate Microparticles on Hela Cells. World J. Nano Sci. Eng. 2012, 2, 25-31. [CrossRef]

17. Kamba, A.S.; Ismail, M.; Azmi Tengku Ibrahim, T.; Zakaria, Z.A.B. Biocompatibility of Bio Based Calcium Carbonate Nanocrystals Aragonite Polymorph on Nih 3 T3 Fibroblast Cell Line. Afr. J. Tradit. Complement. Altern. Med. 2014, 11, 31-38. [CrossRef]

18. Kuusisto, J.E.; Maloney, T.C. The Effect of Carbonation Conditions on the Properties of Carbohydrate-Calcium Carbonate Hybrid Pigments. BioResources 2015, 10, 3277-3292. [CrossRef]

19. Yu, J.; Lei, M.; Cheng, B.; Zhao, X. Effects of PAA Additive and Temperature on Morphology of Calcium Carbonate Particles. J. Solid State Chem. 2004, 177, 681-689. [CrossRef]

20. Westin, K.J.; Rasmuson, A.C. Precipitation of Calcium Carbonate in the Presence of Citrate and EDTA. Desalination 2003, 159, 107-118. [CrossRef]

21. De Jong, W.H.; Borm, P.J.A. Drug Delivery and Nanoparticles: Applications and Hazards. Int. J. Nanomed. 2008, 133-149. [CrossRef]

22. Betancourt, T.; Brannon-Peppas, L. Micro-and Nanofabrication Methods in Nanotechnological Medical and Pharmaceutical Devices. Int. J. Nanomed. 2006, 483-495. [CrossRef]

23. Yadav, K.S.; Chuttani, K.; Mishra, A.K.; Sawant, K.K. Effect of Size on the Biodistribution and Blood Clearance of EtoposideLoaded PLGA Nanoparticles. PDA J. Pharm. Sci. Technol. 2011, 65, 131-139. [PubMed]

24. Jennings, L.; Ivashchenko, O.; Marsman, I.J.C.; Laan, A.C.; Denkova, A.G.; Waton, G.; Beekman, F.J.; Schosseler, F.; Mendes, E. In Vivo Biodistribution of Stable Spherical and Filamentous Micelles Probed by High-Sensitivity SPECT. Biomater. Sci. 2016, 4, 1202-1211. [CrossRef]

25. Sha, X.; Guo, J.; Chen, Y.; Fang, X. Effect of Phospholipid Composition on Pharmacokinetics and Biodistribution of Epirubicin Liposomes. J. Liposome Res. 2012, 22, 80-88. [CrossRef]

26. Aizenberg, J.; Lambert, G.; Weiner, S.; Addadi, L. Factors Involved in the Formation of Amorphous and Crystalline Calcium Carbonate: A Study of an Ascidian Skeleton. J. Am. Chem. Soc. 2002. [CrossRef]

27. Zhao, J.; Wang, M.; Lababidi, H.M.S.; Al-Adwani, H.; Gleason, K.K. A Review of Heterogeneous Nucleation of Calcium Carbonate and Control Strategies for Scale Formation in Multi-Stage Flash (MSF) Desalination Plants. Desalination 2018, 75-88. [CrossRef] 